\title{
WILEY-VCH
}

DOI: 10.1002/ ((please add manuscript number))

Article type: Full Paper

\section{Structural and optical properties of gold/silica "mushroom" particles prepared by interfacial templating}

Szilárd Pothorszky, Dániel Zámbó and András Deák*

Sz. Pothorszky, D. Zámbó, Dr. A. Deák

Institute for Technical Physics and Materials Science, HAS Centre for Energy

Research, P.O. Box 49, H-1525 Budapest, Hungary

E-mail: deak@mfa.kfki.hu

Keywords: gold nanoparticle, Janus particle, single particle spectroscopy

Complex shaped nanoparticles featuring structural or surface chemical patchiness are of special interest in both fundamental and applied research areas. Here we report the preparation and optical properties of gold/silica "mushroom" nanoparticles, where a gold particle is only partially covered by the silica cap. The synthetic approach allows precise control over the particle structure. The interfacial preparation method relies on partially embedding the gold particles in a polystyrene layer that masks the immersed part of the gold particle during silica shell growth from an aqueous solution. By adjusting sacrificial polystyrene film thickness and silica growth time, precise control over the coverage and cap thickness can be achieved. Correlative electron microscopy and single particle scattering spectroscopy measurements underline the high precision and reproducibility of the method. The good agreement between the measured and simulated single particle spectra supported by near-field calculations indicates that the observed changes in the dipolar plasmon resonance are influenced by the extent of coverage of the gold core by the silica cap. The straightforward methods readily available for gold and silica surface modification using range of different (bio)molecules make these well-defined nanoscale objects excellent candidates to study fundamental processes of programmed self-assembly or application as theranostic agents. 


\section{WILEY-VCH}

\section{Introduction}

Hierarchical structures consisting of nanoscale building blocks - especially anisotropic nanoparticles - have attracted much attention due to diverse physicochemical features associated with a single object. ${ }^{[1-4]}$ The broken symmetry of the individual particles makes them suitable candidate for a range of largescale application, such as switchable display devices, ${ }^{[5]}$ interface stabilizers ${ }^{[6]}$ or antireflective coatings. ${ }^{[7]}$ The individual particles on the other hand can take advantage of temperature or electric field gradients that will turn them into nanoscale self-motile objects. ${ }^{[8]}$ For prospective applications, they have already demonstrated their potential in the field of catalysis, ${ }^{[9]} \mathrm{drug}$ delivery $^{[10,11]}$ and optical sensors. ${ }^{[12-14]}$

With the development of advanced nanoparticle synthesis techniques, nanoparticles featuring shape and surface chemistry anisotropy (patchy particles) are intensively being used in more fundamental studies regarding self-assembly as well. ${ }^{[15-18]}$ Both the morphology and the surface heterogeneity of the Janus particles has a profound impact on the assembly process. Chemical patchiness for instance can predefine the selfassembly of the building blocks, eventually leading even to the formation of responsive and reversible superstructures. ${ }^{[19]}$ On the other hand, the complexity of the surface chemistry combined with the non-centrosymmetric geometry of the same particle can enable the preparation of complex structures such as dimers, trimers or helices. ${ }^{[20]}$ The synthesis and directed assembly of Janus particles consisting of organic soft matter and polymers is a rapidly developing field as discussed in many recent excellent reviews (e.g. Refs. ${ }^{[15,21]}$ ). Generally, the size of the polymeric Janus particles is closer to the micron range. Inorganic Janus particles, especially multicompartment particles partly composed of noble metals still has many challenges. Bulk synthesis of plasmonic Janus particles has been successfully carried out e.g. by relying on interfacial nucleation of gold onto polymeric sub-micron particles ${ }^{[22]}$ or competitive ligand coordination 


\section{WILEY-VCH}

combined with silica cap growth, resulting in acorn-like $\mathrm{SiO}_{\mathrm{x}}-\mathrm{Au}$ particles. ${ }^{[23,24]}$ Magnetic-plasmonic dumbbell particles have also been prepared in bulk for simultaneous magnetic and optical detection. ${ }^{[25,26]}$ Similar Janus particles can be also obtained by relying on interfacial films of nanoparticles e.g. evaporating a thin layer of gold on a silica particle monolayer with successive annealing and growth, ${ }^{[27]}$ or by partial entrapping silica particles in a polymer matrix, followed by gold seed particle adsorption at the exposed surface. ${ }^{[28]}$ The presence of the solid interface was used by Miyanohata et al. to hinder the growth of a complete silica shell around gold nanoparticles, leading to an asymmetric particles structure since silica cannot be deposited at the substrate-particle contact point. ${ }^{[29]}$ Nevertheless, fine control over the shell coverage remains a challenge, for which partial masking of the core particle surface is necessary.

The focus of the present study is the development of hybrid silica-gold nanoparticles with mushroom-like Janus morphology, with excellent control over the resulting particle geometry. Symmetry breaking is achieved by embedding the gold nanoparticles in a polymer layer with well-defined thickness at an interface enabling partial exposure of the particle surface during wet-chemical silica shell growth. The synthesis procedure can be extended for the preparation of Janus particles with various shell thicknesses and core diameters. It provides an effective approach to produce asymmetric building blocks for colloidal molecules.

\section{Results and discussion}

In order to realize the preparation strategy outlined in Scheme 1, stable AuNPs in organic solvents with lower dielectric constant such as EtOH and toluene are required. This can be effectively achieved by PEGylation of the nanoparticles using mPEG-SH of appropriate molecular weight. Minimum three centrifuging/redispersing cycles were necessary to minimize water content in the alcosol, which otherwise leads to phase 


\section{WILEY-VCH}

separation when mixed with the PS@toluene solution and also leads to an inhomogeneous PS layer (see Figure S1 of the Supporting Information).

a)

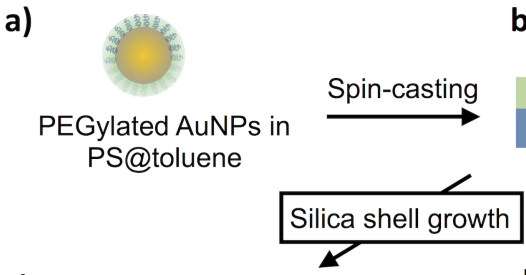

b)

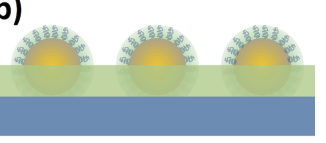

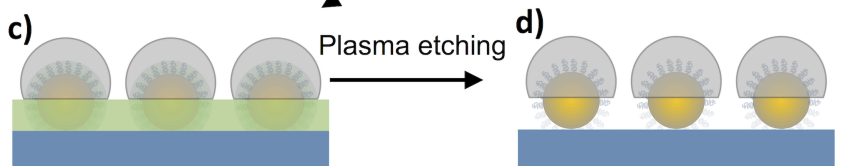

Scheme 1. Schematic illustration of the multistep synthesis procedure. Spin coating of AuNPs in PS@toluene solution (a) results in a submonolayer of gold nanoparticles partially embedded in the polystyrene film on the substrate (b). Silica shell growth is carried out by submerging the whole substrate in the silica growth solution (c) and finally the sacrificial PS layer is removed by plasma etching (d).

\subsection{Verification of the preparation process}

The feasibility of the preparation approach is demonstrated by the SEM images recorded after each consecutive preparation step for the system consist of $66 \mathrm{~nm}$ AuNPs covered by $25 \mathrm{~nm}$ silica shell presented in Figure 1. As shown in Figure 1a, the toluene sol containing a certain ratio of PS and AuNPs is successfully spincoated onto a Si or glass substrate. The presence of the PS also promoted separation of the individual AuNPs, which otherwise (when spin-coated from a pure solvent in high number concentration) tend to form small 2D aggregates under the action of capillary forces. The as-prepared sample was immersed in a silica growth solution for 24 hours in this case. TEOS is known to easily deposit on PEG covered gold nanoparticle surfaces, leading to the formation of a well-defined, compact silica shell. ${ }^{[30]}$ Performing the polycondensation reaction in the given system allows the site-selective deposition of silica at the exposed part of the nanoparticles, where the protective PS layer at the lower part of the particles hinders silica growth. The inset of Figure 1b shows a top-view image of a single particle; the presence of a well-defined, compact silica shell can be clearly observed. Due to the shell growth process the size of objects 


\section{WILEY-VCH}

in the top-view SEM pictures increased from 66 to $116 \mathrm{~nm}$. When considering removal of the sacrificial PS layer, dissolution using toluene would be a possible approach.

However, we used plasma etching to efficiently remove the organic film. The main advantages of plasma-etching in the present case is that it allows to preserve the original particle positions (particle-particle separation and particle orientation), which provides the basis for the correlative single particle spectroscopy, scanning electron microscopy and optical simulation studies. Solvent assisted removal and re-deposition e.g. by drop casting leads to a less defined sample (random particle position/orientation) and significant particle loss (see later below).
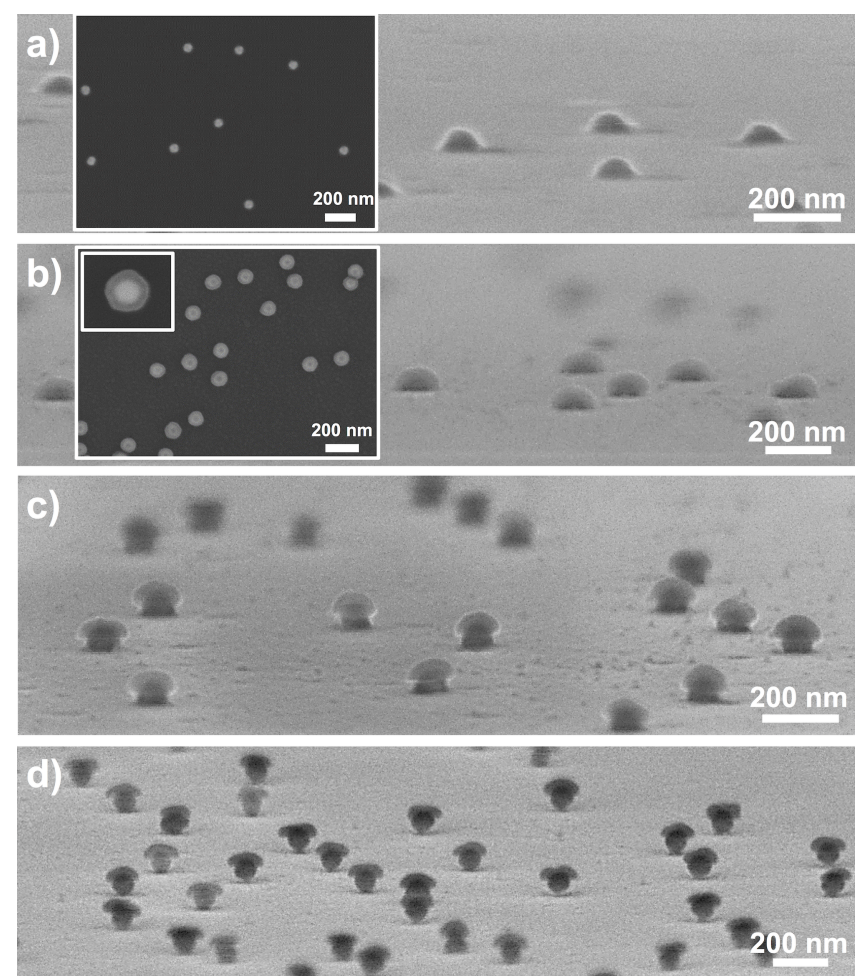

Figure 1. Side-view scanning electron microscopy images of the particles recorded at different stage of the preparation process: directly after spin-coating (a), the silica shell growth (b), and after plasma etching for 1 and 3 minutes (c and d, respectively). The top-view insets in (a) and (b) show the presence of the silica shell after the growth process. Coverage: $33 \%$, shell thickness: $25 \mathrm{~nm}$.

Optimizing the duration of the etching allows to eliminate the PS layer without affecting the Janus nanoparticles. Figure 1c and d show that the etching process itself can be controlled to some extent simply by the duration of the plasma treatment. It is 


\section{WILEY-VCH}

important to point out, that this finding enables the preparation of nanoparticles with well-defined multiple patches by surface modification or further gold synthesis on the newly exposed surface of the core particle. ${ }^{[28]}$

\subsection{Fine-tuning the silica shell geometry (coverage and thickness)}

Two possible strategies can be followed to control the coverage of the gold particle by the silica shell, both rely on fine-tuning the thickness of the polystyrene film that provides the mask during the silica shell growth.

The first strategy involves plasma treatment before performing the silica growth step (corresponding SEM images are shown in the Supporting Information Figure S2). This approach seems to be justified especially observing the as-deposited submonolayer directly after spin-coating (Figure 1a). The SEM images indicate that the surface of the gold nanoparticles above the PS film is not perfectly free of PS, but there might be also a small amount of PS present in this region as indicated by the relatively smooth contours between the planar PS film and the particle surface. Considering the plasma treatment, etching duration is the main control parameter. With increasing etching time, the thickness of the PS film decreases, however, it results in a heterogeneous, less uniform layer. Additionally, heating of the sample in this case is inevitable, that might also affect particle position or orientations once the temperature reaches the glass transition temperature. ${ }^{[31]}$ Still, the most important detrimental consequence is that the relatively intensive plasma treatment not only etches the PS film, but also removes the PEG from the particle surface. This results in a less developed silica cap at after moderate (30 seconds) treatment, but as the thickness of the PS layer is significantly decreased after 2 minutes of etching, the resulting silica shell is less defined and silica deposition also proceeds underneath the particles directly at the substrate level, most probably due to the more efficient PS removal around the particle contours. 


\section{WILEY-VCH}

The second, more effective strategy relies on spin-coating parameter control, such as the polystyrene concentration in the initial casting solution and the spinning speed. By decreasing the concentration of PS, film thicknesses below $50 \mathrm{~nm}$ can be achieved

(Table 1). In order to obtain $25 \mathrm{~nm}$ film thickness, increasing of the spinning speed to $1700 \mathrm{rpm}$ was necessary to preserve homogeneity of the polystyrene thin film.

Table 1. Summary of the spin-coating parameters to control the PS film thickness and the resulting silica cap coverage.

\begin{tabular}{ccccc}
$\begin{array}{c}\text { PS } \\
\begin{array}{c}\text { Concentration } \\
(\mathrm{w} / \mathrm{w} \%)\end{array}\end{array}$ & $\begin{array}{c}\text { Spinning } \\
\text { speed } \\
(\mathrm{rpm})\end{array}$ & $\begin{array}{c}\text { Spinning } \\
\text { time}(\mathrm{sec})\end{array}$ & $\begin{array}{c}\text { PS layer } \\
\text { thickness } \\
(\mathrm{nm})\end{array}$ & $\begin{array}{c}\text { Coverage } \\
\text { by the cap } \\
(\%)\end{array}$ \\
\hline 1 & 1500 & 20 & 50 & 33 \\
0.5 & 1500 & 20 & 33 & 50 \\
0.5 & 1700 & 20 & 25 & 62 \\
\hline
\end{tabular}

Figure 2a-c shows that controlling the PS film thickness provides an effective and convenient way to fine-tune the coverage of the particles. It has to be pointed out, however, that the final shape of the silica cap is strongly correlated to the coverage of the gold particle. As the coverage of the particle increases, the silica cap gets more truncated, leading to the most symmetric shell when the coverage is around $50 \%$ (Figure 2b). For lower and higher core exposure (Figure 2a-c) slightly asymmetric silica coverage was found, that might be attributed to the nucleation site determined anisometric growth at the solid/liquid interface. By using polystyrene of higher molecular weight, PS film thickness values are down to a few nanometre and hence minimal gold particle exposure should be achievable. ${ }^{[32]}$ Besides the coverage of the core particle, controlling the silica cap growth can offer an additional way to fine-tune the final particle morphology. This can be realized by adjusting the contact time of the partially protected particles with the silica growth solution. As the reaction time increased under otherwise the same conditions, increasing silica cap size can be 


\section{WILEY-VCH}

observed (Figure 2d-f). Starting from the same sample, "mushroom" particles featuring cap thickness values of ca. 10, 25 and $60 \mathrm{~nm}$ could be obtained by using 4, 24 and 72 hours contact times, respectively. The resulting cap thickness values show $\pm 5 \%$ or less standard deviation for every sample.
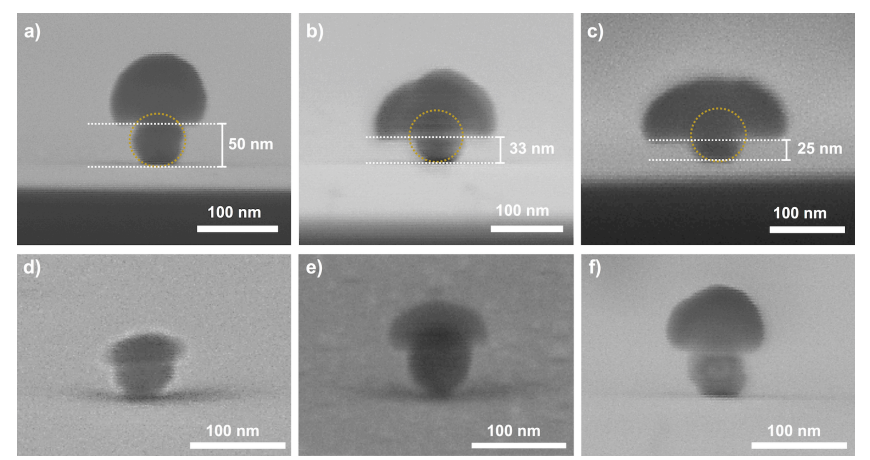

Figure 2. SEM pictures showing the side-view of the "mushroom" particles. Different cap coverage was acieved by changing the thickness of the polystyrene layer: $50 \mathrm{~nm}$ (a), $33 \mathrm{~nm}$ (b) and $25 \mathrm{~nm}$ (c). Different shell thicknesses achieved by adjusting the contact time with the growth solution: 4 (d), 24 (e) and 72 hours (f).

The process can be easily implemented for larger core-particle sizes as long as they remain stable in the toluene solution. Results obtained for smaller (50 $\mathrm{nm}$ diameter) gold nanoparticles (see Supporting Information Figure S3.) indicate that with decreasing core-particle size the morphology of the silica caps shows higher shape and thickness variation, whereby the coverage ratio of the core particles remains highly uniform. This might be attributed to the decreasing size of the nucleation sites available during the silica growth. This finding suggest that a lower size limit might exist using this method for the preparation of well-defined silica caps.

\subsection{Optical properties of the "mushroom" particles}

For any usage of the final Janus particles, they should be recovered from the substrate e.g. by sonication assisted redispersion in a solvent like ultrapure water. During the plasma etching process, however, the silica cap has a protecting effect only on the coated hemisphere and the mPEG-SH in the lower part of the particles gets also degraded. Hence, straightforward recovery of the particles is only possible when a 


\section{WILEY-VCH}

post-synthetic PEGylation was performed out. This was implemented by soaking the substrate with the particles into the aqueous solution of the same mPEG-SH moiety that was used during the particle synthesis. Sonicating enables then recovery of the particles. Despite the low number density of the particles at the substrate, a very weak - but measureable - optical signal can be recorded (Figure 3a). Compared to the starting, PEGylated gold nanoparticles, the extinction spectrum reveals a clear redshift of the localized plasmon resonance peak owing to the higher refractive index of the asymmetric silica cap. ${ }^{[33]}$ Simulation data also confirm that the redshift can be attributed to the presence of a silica shell (see Supporting Information Figure S4). The SEM image recorded by drop-casting the sample confirms the presence of the silica cap after the redispersion process (Figure 3b).
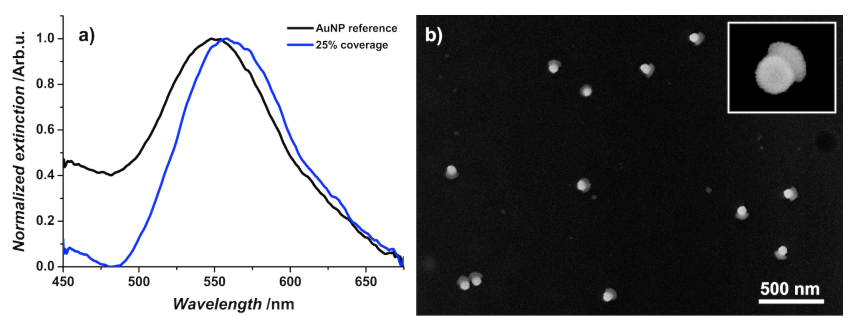

Figure 3. Ensemble optical spectra recorded in water of the PEGylated reference particles and the silica coated "mushroom" particles after sonication aided removal from the substrate (a). SEM images of the "mushroom" particles after drop-casting from aqueous solution. The inset shows a magnified image of the particles clearly showing the presence of the silica cap (b). Coverage: $33 \%$, shell thickness: $25 \mathrm{~nm}$.

To be able to perform correlated optical and scanning electron microscopy measurements and hence more direct information on the structure-optical properties relationship, single particle characterization was carried out on the nanoparticles located at the substrate. Janus particles with two different geometries have been investigated, where the coverages are 33 (Figure 4a) and 50\% (Figure 4b), respectively. Correlated SEM investigations enable that only the optical signal originating from well separated and well defined single particle scatterers are taken into account (Figure 4cf). The averaged single particle spectra are shown in Figure $4 \mathbf{g}$ and reveal a clear 


\section{WILEY-VCH}

difference in the scattering spectra depending on silica cap morphologies. Compared to the reference sample (Au core particle, having been processed in the same way as the capped particles but skipping the silica coating step), the presence of the shell induces a 20 and $27 \mathrm{~nm}$ redshift of the dipole resonance peak for the 33 and $50 \%$ coverages, respectively. The higher redshift observed for the $50 \%$ covered particle can be attributed to the higher coverage of the core particle.
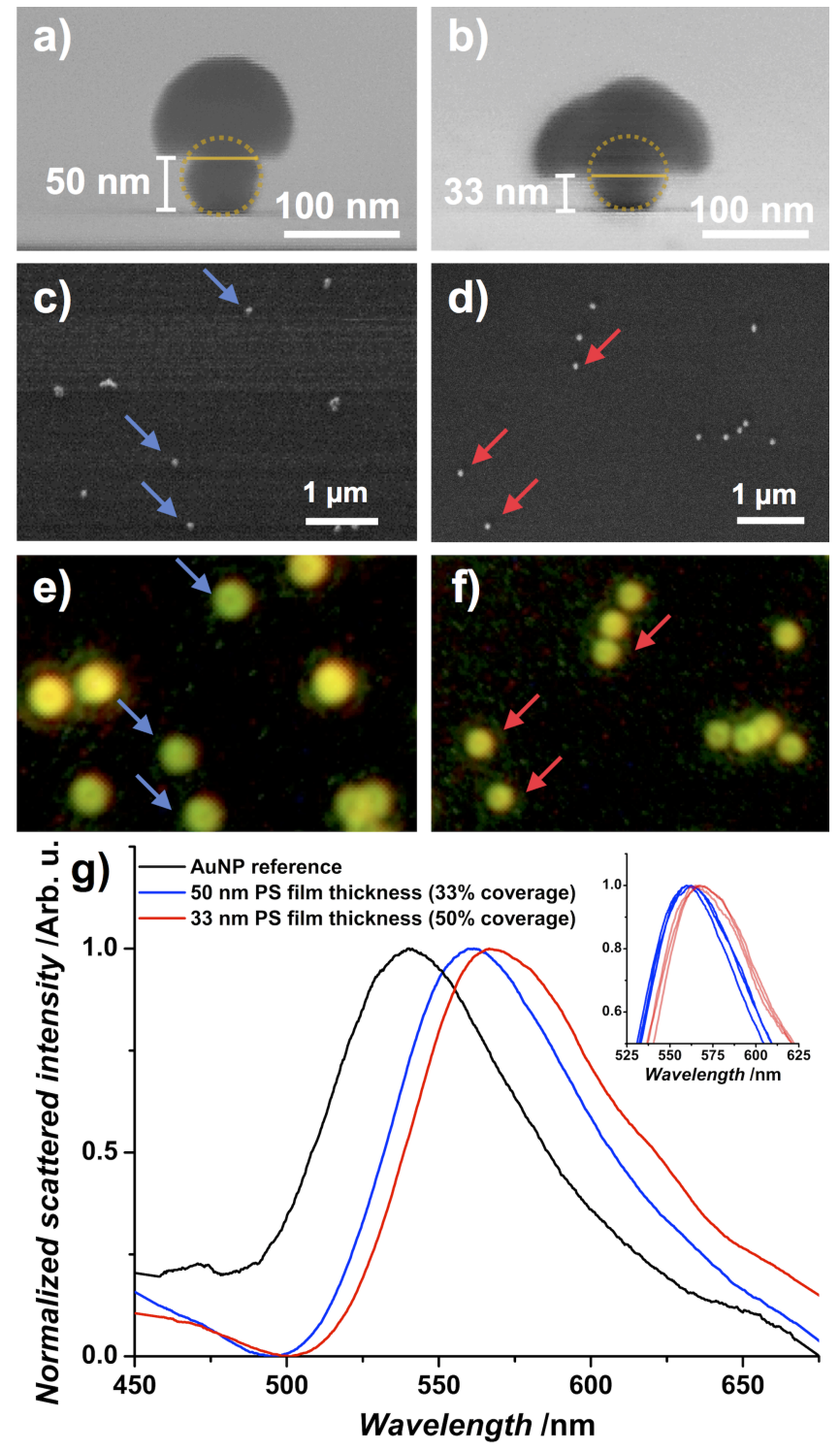

Figure 4. Side-view (a,b) and top-view (c,d) SEM images of the measured particles using 33 and $50 \mathrm{~nm}$ polystyrene film thicknesses. Dark field scattering optical images $(\mathrm{e}, \mathrm{f})$ of the same areas. Arrows mark the individual particles used to obtain single particle scattering spectra. Averaged and normalized single particle scattering spectra (g). A plain PEGylated nanoparticle of the same size after the same process is also shown as reference. The inset shows the magnified region around the resonance peak for each individual spectra. 


\section{WILEY-VCH}

The inset in Figure $\mathbf{4 g}$ shows a magnified view of the individual resonance peaks that were obtained for the particles marked by the arrows in Figure 4c-f. For a given particle type there is only a minor variation in the scattering spectrum. This on the one underlines the size/shape homogeneity of these particles, on the other it also shows that the key parameter determining the optical response is indeed the different silica cap morphology.
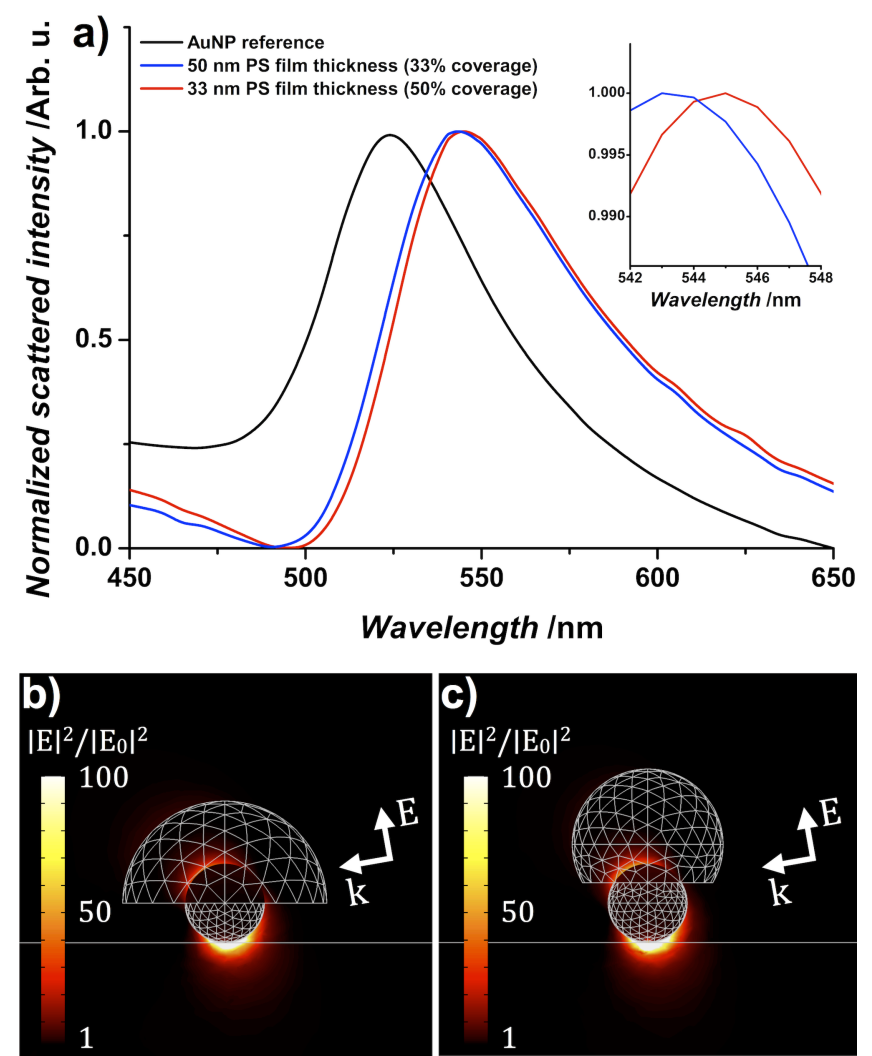

Figure 5. Simulated single particle spectra obtained for the reference particles (no coating), and the particles having 50 and 33\% core-particle coverage by the silica cap after normalization (a). Near-field intensity maps obtained at their respective resonance peak maxima for the particles with 50 (b) and 33\% (c) coverage. The inset shows the magnified region around the resonance. Angle of incidence: $80^{\circ}$; substrate refractive index: 1.52 .

The role of the silica cap is also verified by the simulation data. Figure 5a shows the simulated scattering spectra for the two particle geometries, together with the reference spectrum. During the calculations only TM excitation (p-polarized illumination) was taken into account, since its contribution to the scattering cross-section is about 2 orders of magnitude larger compared to the TE excitation (s-polarized illumination). 


\section{WILEY-VCH}

Additionally, using the current experimental setup, the contribution of the p-polarized light to the excitation is also about an order of magnitude higher. ${ }^{[34]}$ The observed redshift of the two resonance peaks measures 18 and $20 \mathrm{~nm}$, respectively. Comparing the measured and simulated spectra two main conclusion can be drawn. First, there is a qualitative agreement between the measured and simulated spectral shifts due to the silica cap, in both cases thicker silica shell causes a larger redshift of the spectrum. Second, the actual peak-position values for all particle types are found at longer wavelength than for the measurements.

For the first, the qualitative agreement between the experimental and theoretical shift can be attributed to the well-defined structure of "mushroom" particles due to the preparation process. The difference between the two peak positions corresponding to the two different particle types is rather small. This is in agreement with earlier simulation results on similar particle geometries, which also indicate that the extent of redshift due to partial silica coating is rather modest. ${ }^{[35]}$ Our results can be well explained based on the developing near-field distribution around the particles due to dark-field illumination (Figure 5b,c). For the given experimental setup, dark-field excitation impinges on particles at a large angle of incidence $\left(\sim 80^{\circ}\right)$. Using this angle of incidence in the calculation, a strong interaction with the substrate develops, leading to a large field enhancement in the region underneath the particles. ${ }^{[36]}$ In the cap region, however, most of the near field effectively decays over the distance covered by the silica shell thickness. Hence, coverage will play the determining role in the redshift, while the silica shell thickness should not influence the peak position due to the large angle of incidence on the other hand, already at $33 \%$ coverage most of the near-field decays within the shell, and the increase to $50 \%$ will have only a small effect compared to the substrate. 


\section{WILEY-VCH}

For the second, the systematic redshift of the experimental data suggests that the actual medium around the particles might possess higher refractive index as anticipated. It can be speculated, that underneath the particles trace amount of PEG, polystyrene or their degradation product is present due to the incomplete removal by the plasma etching. Capillary condensation might be the other reason for a higher dielectric constant in this region, ${ }^{[37]}$ since the scattering spectra were collected under ambient condition. These factors might be also responsible for the larger measured spectral difference between the two different coverages when compared to the simulation results. More detailed and targeted investigations are needed to clarify the origin of the systematic redshift.

\section{Conclusion}

In the present work gold nanoparticles partially covered by a silica cap have been prepared and characterized by correlative scanning electron microscopy and single particle spectroscopy. The partial coverage was achieved via interfacial templating, by protecting part of the gold surface by a polystyrene thin film, that was effectively masking the immersed part of the particles during solution based silica shell growth. The presented approach allows the preparation of particles with well-defined geometry. Precise control over the resulting particle geometry, that is coverage and silica shell thickness can be achieved by fine-tuning the polystyrene film thickness and the silica shell growth time. The optical spectrum of the particles can be tuned by the parameters of the silica shell; a good agreement between single particle scattering spectra and finite element simulation data was found. Due to the well-defined structure of the particles, and readily available well-established protocols for gold and silica surface modification, the prepared particles are excellent candidates for fundamental studies in programmed self-assembly of patchy nanoparticles at the nanoscale. Due to the gold core, optical readout of the assembly process should be feasible relying on the well- 


\section{WILEY-VCH}

known optical effects of plasmon coupling and resonance shift monitoring. Decoration of the hybrid particles with biological and/or fluorophores can find also application in the field of theranostics. 


\section{WILEY-VCH}

\section{Experimental}

\section{Materials}

Sodium citrate tribasic dihydrate (ACS reagent, $\geq 99 \%$ ), cetyltrimethylammonium bromide (CTAB, 99\%), sodium borohydride $\left(\mathrm{NaBH}_{4}, 99 \%\right)$, L-ascorbic acid (AA, $>99 \%$ ), potassium iodide (KI, >99\%), tetrachloroauric acid trihydrate $(99.9 \%)$, tetraethylorthosilicate (TEOS, 98\%), ammonium hydroxide solution $\left(\mathrm{NH}_{3}\right.$, ACS reagent 28-30\%), toluene (CHROMASOLV®, for HPLC, 99.9\%), polystyrene $(280 \mathrm{kDa})$ were purchased from Sigma-Aldrich. mPEG-SH (5kDa) was obtained from Rapp Polymere GmbH. Ethanol (EtOH, absolute) were purchased from VWR. Synthesis and PEGylation of the AuNPs

Gold NPs with the diameter of $66 \mathrm{~nm}$ were prepared according to a seed-mediated protocol. ${ }^{[38]}$ For PEGylation, $20 \mathrm{ml}$ of the as-prepared sol was treated with mPEG-SH (0.1 $\mathrm{g}$ in $1 \mathrm{ml}$ Milli-Q water) for $2 \mathrm{~h}$. In the next step, the PEGylated particles were purified with three cycles of centrifugation (10 min, $3000 \mathrm{rcf})$ and redispersion in 20 ml EtOH.

\section{Submonolayer preparation and site-selective silica coating}

The PEGylated AuNPs dispersed in EtOH were transferred into 0.5 or $1 \mathrm{w} / \mathrm{w} \%$ PS in toluene. This was spin-casted on a Si or glass substrate. Spin parameters were optimized so that the thickness of the resulting PS layer was smaller than the diameters of the AuNPs (see Results and Discussion part for details). The substrate with the partially masked gold particles was immersed in a silica growth solution consisting of $3.7 \mathrm{ml} \mathrm{EtOH}, 0.9 \mathrm{ml}$ Milli-Q water; $0.067 \mathrm{ml} \mathrm{NH}_{3}$ and $0.1 \mathrm{ml} \mathrm{1 \%} \mathrm{TEOS} \mathrm{in} \mathrm{EtOH.} \mathrm{The}$ concentration of the TEOS was set to be under the critical value of homogeneous nucleation. ${ }^{[39]}$ The silica cap growth was allowed to proceed 4, 24 or 72 hours in order to produce different $(10,30$ and $60 \mathrm{~nm})$ shell thickness. The substrate was rinsed with 


\section{WILEY-VCH}

EtOH and dried in a nitrogen stream. The last step was the removal of the polystyrene film by performing plasma treatment for 3 min (Harrick PDC-32G).

\section{Characterization techniques}

Scanning electron microscope (SEM) images were obtained using Zeiss LEO field emission scanning electron microscope operated at $5 \mathrm{keV}$ acceleration voltage. Ensemble optical spectra in solvent were measured by a Thorlabs CCS200 fibre coupled VIS spectrometer. Single particle spectroscopy was carried out using a custom built setup, consisting of an Olympus BX51 optical microscope combined with an imaging spectrograph (Horiba Jobin Yvon CP140) and a high sensitivity TE-cooled CCD camera (Andor iKON-M). Single particle spectra were recorded using a 100x dark-field microscope objective $(\mathrm{NA}=0.9)$ in epi dark-field illumination mode. Simulated spectra were obtained by finite element method (FEM) using the RF Module of COMSOL Multiphysics (v4.3a) based on the modification and expansion of an existing model (Model ID: 14443). The scatterer particle geometry was constructed based on the sizes obtained from the SEM measurements. The far-field scattering was obtained by restricting the integration of the outward scattered power flux density (Poynting) in the far-field over a spherical surface to an area which is defined by our numerical aperture $(\mathrm{NA}=0.9)$, that is by the collection cone of the objective. The obtained scattered power is divided by the monitor (integration) area to obtain scattered intensity. This is normalized between 0 and 1 and serves as the input data for Figure 5a. The p-polarized (TM-mode) plane wave illumination was implemented at $80^{\circ}$ angle of incidence; substrate refractive index of 1.520 was used (no substrate absorption).

The dielectric function of gold was obtained from Olmon et. al. ${ }^{[40]}$

\section{Supporting Information}

Supporting Information is available from the Wiley Online Library or from the author.

\section{Acknowledgements}




\section{WILEY-VCH}

We thank Enikő Traply for her assistance in the preparative and László Bicsérdy and Zoltán Hajnal in the simulation work. The project was supported by the Hungarian Scientific Research Fund OTKA-PD-105173 and K-112114. A. D. acknowledges the support of the János Bolyai Research Fellowship from the Hungarian Academy of Sciences. Sz. P. and D. Z. acknowledge the support of the Pro Progressio Foundation and József Varga Foundation.

\section{Notes and references}

[1] A. Perro, S. Reculusa, S. Ravaine, E. Bourgeat-Lami, E. Duguet, Journal of Materials Chemistry 2005, 15, 3745.

[2] A. Walther, A. H. E. Müller, Chemical Reviews 2013, 113, 5194.

[3] S. C. Glotzer, M. J. Solomon, Nature materials 2007, 6, 557.

[4] J. Du, R. K. O’Reilly, Chemical Society Reviews 2011, 40, 2402.

[5] T. Nisisako, T. Torii, T. Takahashi, Y. Takizawa, Advanced Materials 2006, 18, 1152.

[6] A. Walther, K. Matussek, A. H. Muller, Acs Nano 2008, 2, 1167.

[7] H. Y. Koo, D. K. Yi, S. J. Yoo, D.-Y. Kim, Advanced Materials 2004, 16, 274.

[8] W. Wang, W. Duan, S. Ahmed, T. E. Mallouk, A. Sen, Nano Today 2013, 8, 531.

[9] J. Faria, M. P. Ruiz, D. E. Resasco, Advanced Synthesis \& Catalysis 2010, 352, 2359.

[10] J. A. Champion, Y. K. Katare, S. Mitragotri, Journal of Controlled Release 2007, 121, 3.

[11] S. Yang, F. Guo, B. Kiraly, X. Mao, M. Lu, K. W. Leong, T. J. Huang, Lab on a Chip 2012, 12, 2097.

[12] M. D. McConnell, M. J. Kraeutler, S. Yang, R. J. Composto, Nano Letters 2010, 10, 603.

[13] M. Himmelhaus, H. Takei, Sensors and Actuators B: Chemical 2000, 63, 24.

[14] D. Shao, X. Zhang, W. Liu, F. Zhang, X. Zheng, P. Qiao, J. Li, W. Dong, L. Chen, ACS Applied Materials \& Interfaces 2016, 8, 4303.

[15] S. Jiang, Q. Chen, M. Tripathy, E. Luijten, K. S. Schweizer, S. Granick, Advanced Materials 2010, 22, 1060.

[16] B. Fang, A. Walther, A. Wolf, Y. Xu, J. Yuan, A. H. E. Müller, Angewandte Chemie International Edition 2009, 48, 2877.

[17] W. Ma, L. Xu, L. Wang, H. Kuang, C. Xu, Biosensors and Bioelectronics 2016, 79, 220.

[18] C. Shen, X. Lan, X. Lu, T. A. Meyer, W. Ni, Y. Ke, Q. Wang, Journal of the American Chemical Society 2016, 138, 1764.

[19] S. K. Smoukov, S. Gangwal, M. Marquez, O. D. Velev, Soft Matter 2009, 5, 1285.

[20] D. Zerrouki, J. Baudry, D. Pine, P. Chaikin, J. Bibette, Nature 2008, 455, 380.

[21] F. Wurm, A. F. Kilbinger, Angewandte Chemie International Edition 2009, 48, 8412.

[22] Y. Wang, T. Ding, J. J. Baumberg, S. K. Smoukov, Nanoscale 2015, 7, 10344.

[23] T. Chen, G. Chen, S. Xing, T. Wu, H. Chen, Chemistry of Materials 2010, 22, 3826.

[24] C. C. Crane, J. Tao, F. Wang, Y. Zhu, J. Chen, The Journal of Physical Chemistry C 2014, 118, 28134.

[25] H. Yu, M. Chen, P. M. Rice, S. X. Wang, R. L. White, S. Sun, Nano Letters 2005, 5, 379.

[26] H. Gu, Z. Yang, J. Gao, C. K. Chang, B. Xu, Journal of the American Chemical Society 2005, 127, 34.

[27] Y. Lu, H. Xiong, X. Jiang, Y. Xia, M. Prentiss, G. M. Whitesides, Journal of the American Chemical Society 2003, 125, 12724.

[28] C.-C. Lin, C.-W. Liao, Y.-C. Chao, C. Kuo, ACS Applied Materials \& Interfaces 2010, 2, 3185.

[29] R. Miyanohata, T. Matsushita, T. Tsuruoka, H. Nawafune, K. Akamatsu, Journal of Colloid and Interface Science 2014, 416, 147.

[30] C. Fernández-López, C. Mateo-Mateo, R. A. Álvarez-Puebla, J. Pérez-Juste, I. PastorizaSantos, L. M. Liz-Marzán, Langmuir 2009, 25, 13894. 


\section{WILEY-VCH}

[31] A. Bottomley, D. Prezgot, J. P. Coyle, A. Ianoul, Nanoscale 2016, 8, 11168.

[32] D. B. Hall, P. Underhill, J. M. Torkelson, Polymer Engineering \& Science 1998, 38, 2039.

[33] Z. W. Seh, S. Liu, M. Low, S.-Y. Zhang, Z. Liu, A. Mlayah, M.-Y. Han, Advanced Materials 2012, 24, 2310.

[34] L. Jiang, T. Yin, Z. Dong, M. Liao, S. J. Tan, X. M. Goh, D. Allioux, H. Hu, X. Li, J. K. W. Yang, Z. Shen, ACS Nano 2015, 9, 10039.

[35] C. Du, J. Dong, T. Chen, C. He, D. Shi, Plasmonics 2013, 8, 1523.

[36] M. Hu, A. Ghoshal, M. Marquez, P. G. Kik, The Journal of Physical Chemistry C 2010, 114,7509 .

[37] F. Gallego-Gómez, A. Blanco, C. López, Advanced Materials 2015, 27, 2686.

[38] X. Ye, C. Zheng, J. Chen, Y. Gao, C. B. Murray, Nano Letters 2013, 13, 765.

[39] K.-S. Chou, C.-C. Chen, Ceramics International 2008, 34, 1623.

[40] R. L. Olmon, B. Slovick, T. W. Johnson, D. Shelton, S.-H. Oh, G. D. Boreman, M. B. Raschke, Physical Review B 2012, 86, 235147. 


\section{WILEY-VCH}

Janus nanoparticles featuring „mushroom“ like morphology consisting of a gold particle core and a silica cap are fabricated by interfacial templating approach. Excellent control over the silica shell morphology (coverage and thickness) is achieved by partial masking of the core-particle by a polymer thin film during wet-chemical silica growth. The precision of the preparation process is confirmed by correlative scanning electron microscopy and single particle spectroscopy measurements.

\section{Keyword}

interfacial templating, Janus nanoparticle, single particle spectroscopy

Szilárd Pothorszky, Dániel Zámbó and András Deák*

Structural and optical properties of gold/silica "mushroom" particles prepared by interfacial templating

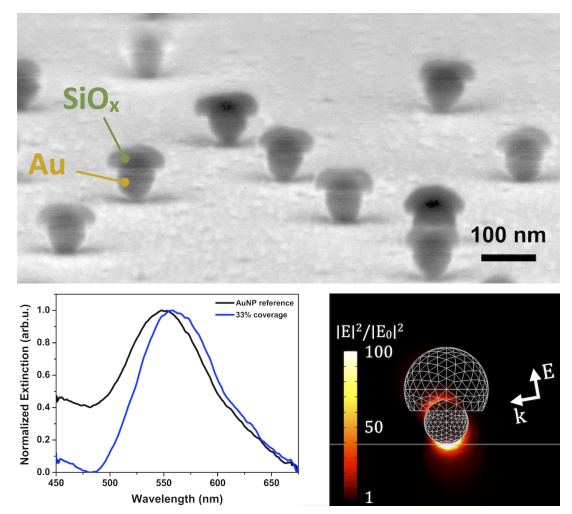

